\title{
Superficial epithelioma with sebaceous differentiation: a case report with literature review
}

\author{
André Coelho ${ }^{\circledR}$, Boštjan Luzar²
}

\begin{abstract}
Superficial epithelioma with sebaceous differentiation is the original name for a rare benign tumor that lacks consensus in the literature, both in nomenclature and in diagnostic criteria. We report the case of a 68-year-old male with a white papule on the left lower eyelid, fully excised. On histology, the lesion was a superficial, lobulated, plate-like epithelial proliferation, with small mature clusters of sebocytes and ductal structures. Mismatch repair proteins MLH-1, MSH-2, MSH-6, and PMS-2 were retained in the immunohistochemistry study. We reviewed the literature and added our case, which we believe contributes to better characterization of superficial epithelioma with sebaceous differentiation. It usually presents as a solitary papule or nodule on the face of middle-aged males. Histologically, besides the hallmark of a superficial plate-like epithelial proliferation, some variations are occasionally reported: the presence of randomly scattered keratin-filled cysts, squamous eddies, minimal peripheral palisading, marked verruciform architecture, and a reticulated pattern with elongated strands of keratinocytes. The mitotic activity can be high, but no cytological atypia or necrosis is seen. If properly removed, there is no evidence of local recurrence or distant spread. The association with Muir-Torre syndrome remains unclear.
\end{abstract}

Keywords: superficial epithelioma with sebaceous differentiation, case report, review, pathology, tumor, Muir-Torre syndrome

Received: 10 July 2017 | Returned for modification: 24 July 2017 | Accepted: 26 July 2017

\section{Introduction}

Superficial epithelioma with sebaceous differentiation (SESD) is a rare histologically distinctive benign tumor, described for the first time in 1980 by Rothko et al. as multiple lesions on a single patient (1). Since then, 20 more cases have been reported, most of them solitary lesions, mainly on the faces or backs of middleaged to elderly persons (2-12). The nomenclature of this tumor has been widely discussed, and the concept of reticulated acanthoma with sebaceous differentiation (RASD) was introduced by Steffen and Ackerman in 1993 in an effort to rename SESD (13). In 1995, Yuz et al. proposed the term sebomatricoma for all benign lesions with sebaceous differentiation, which SESD would fall into (14). Later, in 2007, LeBoeuf et al. considered the importance of maintaining the original nomenclature, although some authors have proposed the term RASD for a distinct lesion from SESD based on the presence of mature sebaceous ductal structures on the former and their complete absence on the latter (8-9). For the sake of clarification, we use the term SESD as originally intended by Rothko et al. and later discuss our view on the nomenclature.

\section{Case report}

We report the case of a 68-year-old male with no relevant clinical history that presented with a longstanding white papule measuring $7 \times 6 \mathrm{~mm}$ on the left lower eyelid. The lesion was fully excised. On histology, the tumor presented as a superficial, lobulated, plate-like epithelial proliferation with multiple broad connections to the epidermis and well-defined pushing borders into the papillary dermis (Fig. 1). The tumor cells were oval with pale basophilic nuclei, visible nucleoli, and scant pale eosinophilic cytoplasm, arranged peripherally in a haphazard distribution without palisad- ing. In the center of the lobules, on the lower portion of the lesion, small mature clusters of sebocytes were seen, with microvesicular cytoplasm and scalloped central nuclei (Figs. 2-3). Sebaceous ductal structures were also clearly seen. The upper central areas had squamous differentiation, with more abundant cytoplasm and occasionally forming squamous eddies. The mitotic activity was focally conspicuous, with 11 mitoses per 10 high-power fields (Fig. 3). The papillary dermis surrounding the tumor displayed dilated blood vessels and a mild lymphocytic inflammatory response (Fig. 4). Immunohistochemistry staining with antibodies for MLH-1, MSH-2, MSH-6, and PMS-2 revealed the absence of loss of nuclear staining in neoplastic cells (Fig. 5).

\section{Discussion}

The clinical presentation of SESD is usually indistinct, most frequently a papule or a nodule on the face of adults $(1-2,4,6,9,12)$. A more distinctive yellow, well-defined plaque has also been described, which seems to better reflect its histological characteristics $(3-4,7,11)$. Unusual features such as pigmentation or a partial halo of hypopigmentation have been reported, and some authors have also described a yellow discharge from the tumor $(2-3,6,8)$. Notably, in the original description by Rothko et al. the patient presented with multiple lesions, but most cases reported afterwards were solitary (1). The clinical features of the published cases are summarized in Table 1 . The age of presentation in patients is wide, ranging from 38 to 85 years old, with a mean age of 63 years, and there is a male predominance, with a male-to-female ratio of 2.14:1. The most frequent location is the face ( $56 \%$ of all cases), followed by the back region (22\%), with only isolated cases on the neck, trunk, flank, gluteal area, axilla, and thigh. The tumor diameter is usually smaller than $20 \mathrm{~mm}$, with a mean size of $9.8 \mathrm{~mm}$. 


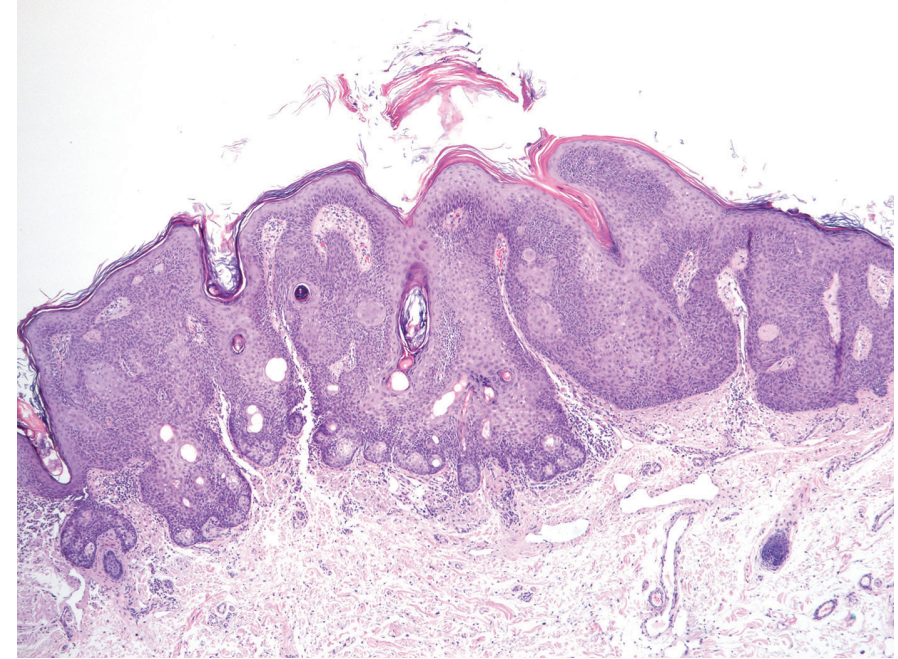

Figure 1 | A superficial, plate-like lobular epithelial proliferation with numerous attachments to the epidermis can be seen (H\&E, $4 \times$ ).

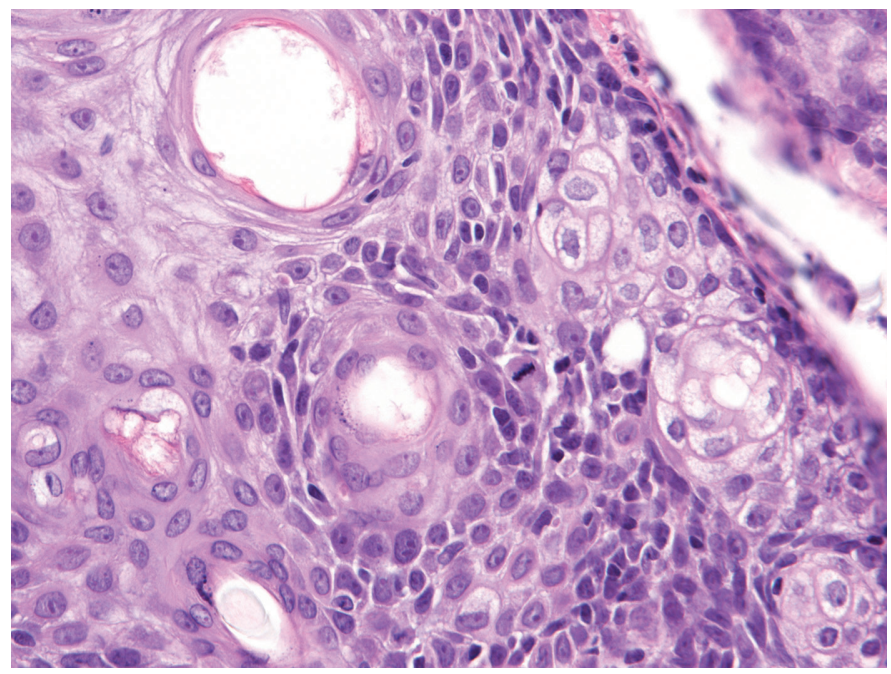

Figure 3 | Sebaceous differentiation is evident. Note the basaloid cells with vesicular nuclei, visible nucleoli, and scant eosinophilic cytoplasm. There is also one mitosis in the center of the field (H\&E, 40x).

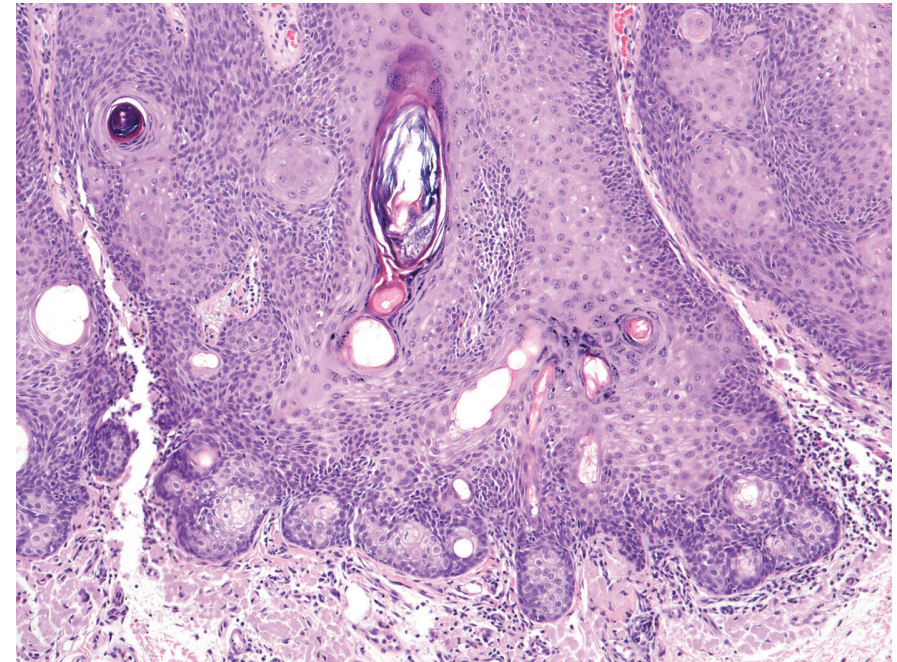

Figure $2 \mid$ On the deep portion of the tumor there are small clusters of mature sebocytes and ductal structures (H\&E, 10x).

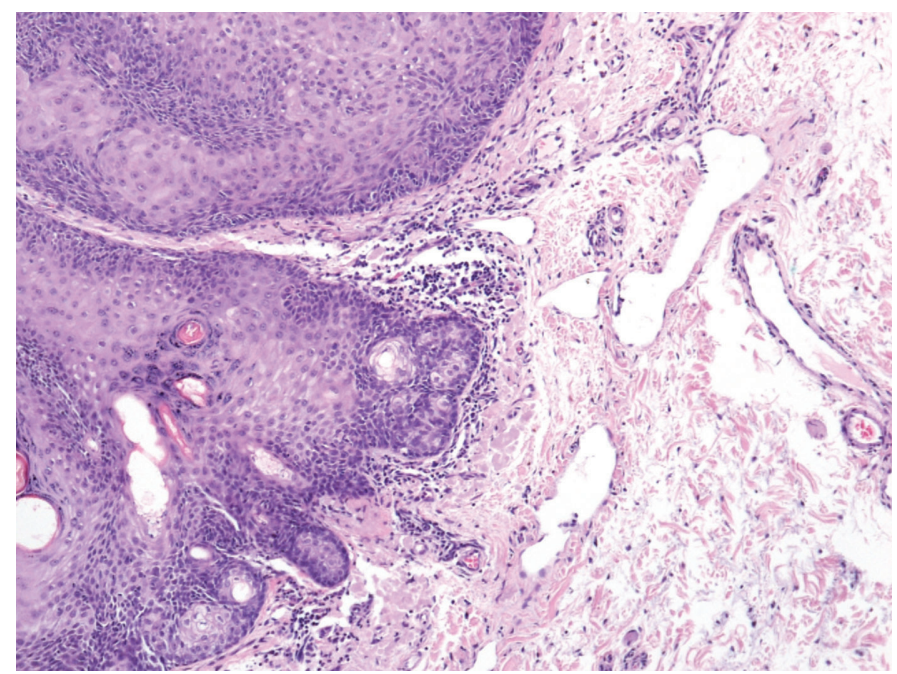

Figure 4 | The dermis shows a mild host response and a few dilated blood vessels (H\&E, 10x).

Table 1 | Summary of clinical features of published cases.

\begin{tabular}{|c|c|c|c|c|c|}
\hline & Author & Age $(y) / \operatorname{Sex}$ & Location & Clinical description & Size $(\mathrm{mm})$ \\
\hline 1. & Rothko $^{1}$ & $48 / \mathrm{M}$ & $\begin{array}{l}\text { multiple (nose, cheek, upper lip, } \\
\text { trunk, thigh, axilla) }\end{array}$ & $3 \times$ papule; $3 \times$ nodule & 3-15 \\
\hline 2. & Friedman ${ }^{2}$ & $66 / F$ & neck & smooth nodule & 10 \\
\hline 3. & Friedman ${ }^{2}$ & $72 / M$ & cheek & erythematous hyperkeratotic papule & 7 \\
\hline 4. & Friedman $^{2}$ & $72 / \mathrm{F}$ & forehead & hyperkeratotic pigmented nodule & 10 \\
\hline 5. & Friedman ${ }^{2}$ & $57 / M$ & eyelid & pearly papule & 8 \\
\hline 6. & Friedman ${ }^{2}$ & $65 / F$ & cheek & pale papule & 4 \\
\hline 7. & Vaughan $^{3}$ & $55 / F$ & back & $\begin{array}{l}\text { yellow, flat-topped, well-defined plaque with a partial halo of } \\
\text { hypopigmentation }\end{array}$ & 14 \\
\hline 8. & Kato $^{4}$ & $38 / \mathrm{F}$ & eyelid & yellow hard plaque & 6 \\
\hline 9. & Yus $^{5}$ & $60 / M$ & gluteal area & erythematous plaque & 45 \\
\hline 10. & Akasaka $^{6}$ & $68 / M$ & cheek & hyperkeratotic pigmented umbilicated nodule & 4 \\
\hline 11. & Lee $^{7}$ & $59 / \mathrm{F}$ & back & yellow, erythematous, flat-topped, well defined plaque & 20 \\
\hline 12. & Fukai $^{8}$ & $55 / \mathrm{F}$ & back & granular, pink to brown plaque, with yellow discharge & 18 \\
\hline 13. & LeBoeuf 9 & $70 / M$ & back & tan lesion & 5 \\
\hline 14. & LeBoeuf ${ }^{9}$ & $61 / \mathrm{M}$ & nose & verrucous lesion & 5 \\
\hline 15. & LeBoeuf ${ }^{9}$ & 79/M & back & $\tan$ lesion & 3 \\
\hline 16. & LeBoeuf ${ }^{9}$ & $45 / M$ & multiple ( $2 \times$ ear) & $2 \times$ dark tan lesion & 6,8 \\
\hline 17. & LeBoeuf ${ }^{9}$ & $79 / M$ & ear & $\tan$ lesion & 4 \\
\hline 18. & LeBoeuf ${ }^{9}$ & $77 / M$ & flank & brown plaque & 15 \\
\hline 19. & Haake $^{10}$ & $85 / M$ & back & erythematous plaque & $\mathrm{n} / \mathrm{a}$ \\
\hline 20. & Shon $^{11}$ & $53 / M$ & back & yellow plaque & 10 \\
\hline 21. & Aldrees $^{12}$ & $48 / M$ & eyelid & tan papule & 4 \\
\hline 22. & Present case & $68 / M$ & eyelid & white papule & 7 \\
\hline
\end{tabular}

F: female; $M$ : male. 


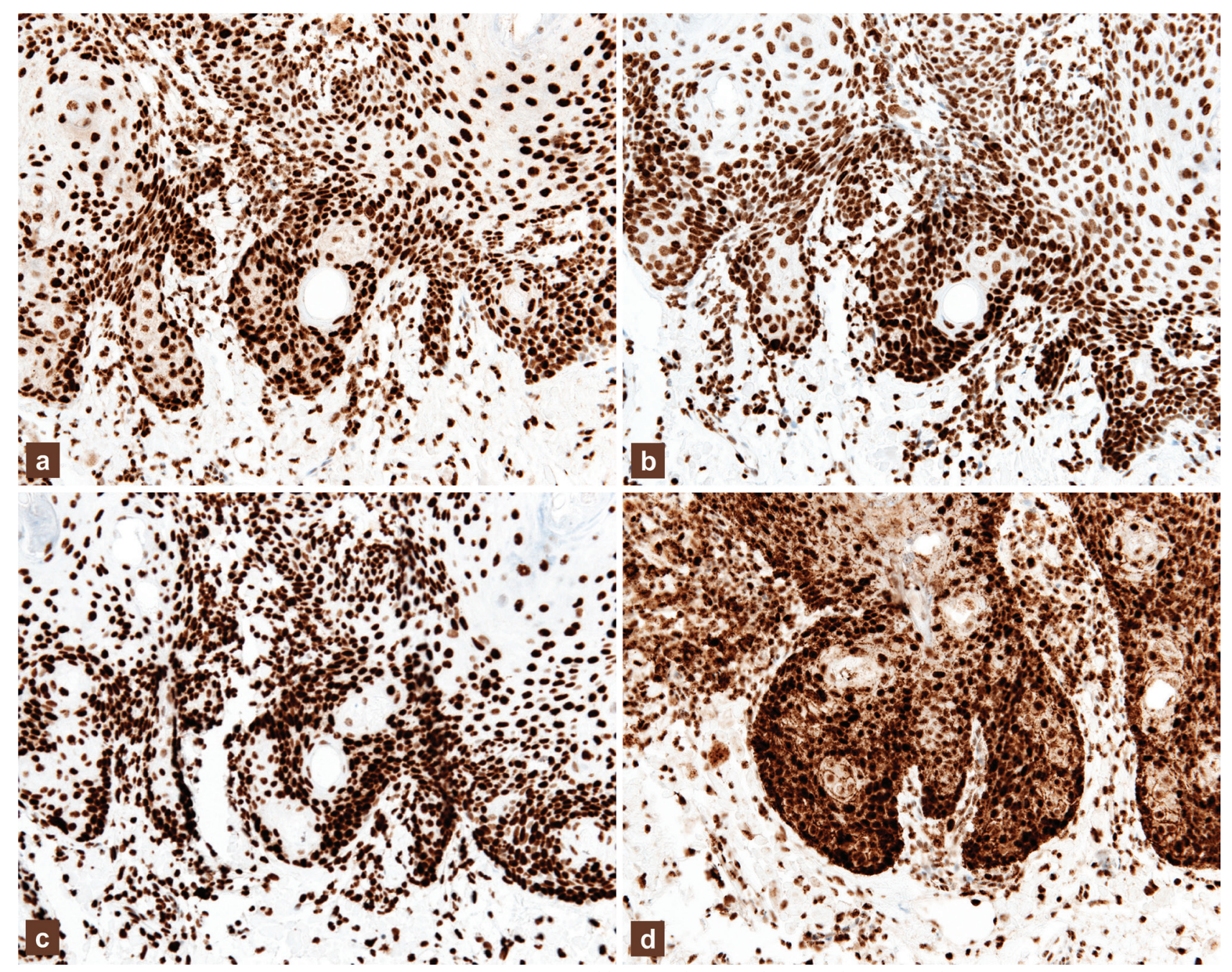

Figure 5 | There is no loss of nuclear staining in the mismatch repair proteins (a) MLH1; b) MSH2; c) MSH6; d) PMS2, 20x).

The tumor is more distinctive on histological examination, featuring a superficial, multilobular, plate-like proliferation with broad connections to the overlying epidermis, well-defined borders, and sebaceous differentiation with characteristic mature sebocytes arranged in small clusters or single cells in the deep portions and periphery of the tumor $(1-6,7,9,12)$. Additional features frequently reported are sebaceous ductal structures, randomly scattered keratin-filled cysts, squamous eddies, and minimal to absent peripheral palisading $(1-9,12)$. The cells are described as uniform, with round to oval or basaloid morphology, scant to moderate eosinophilic cytoplasm, pale basophilic nuclei, and visible but not prominent nucleoli $(1-4,6,7,12)$. A variable host lymphocytic response with mild fibrosis is also frequently reported (1-2, 9-11).

Some architectural differences are reported on otherwise similar lesions, which include two cases with marked verruciform architecture and three cases of a reticulated pattern with elongated strands of keratinocytes (8-11). This latter pattern is responsible for some confusion in the nomenclature because it is reported as SESD or RASD, either as synonyms or as entirely distinct lesions $(8-11,13)$.

Other histological features rarely reported are parakeratosis, the presence of large lobules of sebocytes in the lower part of the tumor that occasionally undergo cystic degeneration, the scattering of individual sebocytes or small clusters throughout the lesion, sebaceous cell clusters occasionally opening onto the skin surface, and increased melanin pigment $(1-6,8)$. One case with the classical morphology but with additional apocrine differen- tiation was considered a distinct entity, reported as reticulated acanthoma with apocrine differentiation (15).

The mitotic activity is variable, usually low, with one report of a case with six mitoses per 10 high-power fields but without any atypical mitosis (2). Our case has the highest mitotic activity reported, with 11 mitoses per 10 high-power fields, but the lesion was otherwise unremarkable, without cytological atypia, necrosis, or atypical mitoses that could warrant a suspicion of malignancy. SESDs are capable of local recurrence if partially excised, as demonstrated by two recurring cases reported by Vaughan et al. and Kato et al., one after 8 months and the other not specified (3-4). If properly removed, no evidence of local recurrence or distance spread was reported in pure cases of SESD $(1-4,6)$. One exceptional case, however, describes a long-standing plaque 45 $\mathrm{mm}$ in diameter, removed after the development of an eccentric nodular area. It was diagnosed as an apocrine adenocarcinoma developed on the background of a SESD, suggesting a malignant potential for this otherwise benign lesion (5)

One case was associated with internal malignancy (2). The patient died from esophageal and colonic carcinoma, and his sister also died with carcinoma in an unknown location. Although the clinical features could suggest Muir-Torre syndrome, the patient was not tested for microsatellite instability. One patient, however, was reported to have SESD in addition to sebaceous adenomas and a significant family history of visceral malignancies, in which further investigation revealed reduced MSH-6 protein expression by immunohistochemistry technique (11). Other case reports did not find evidence of microsatellite instability on SESD, although 
only MSH-2 and MLH-1 were studied (8-10). Our case was tested for MLH-1, MSH-2, MSH-6, and PMS-2, but no loss of protein expression was found. More cases should be tested to determine the possible relation to Muir-Torre syndrome.

Due to its characteristic histologic features, SESD is considered a distinct entity, although some overlapping morphology with sebaceous or epidermal tumors may hinder a precise classification $(1-4,6-12,14,16)$. SESD shares features with sebaceous neoplasms such as nevus sebaceous, sebaceous adenoma, and sebaceoma, and also with other tumors that may display sebaceous differentiation, such as basal cell carcinoma. The plate-like configuration of SESD is also seen in seborrheic keratosis and in the tumor of the follicular infundibulum. However, nevus sebaceous often presents at birth with variable irregularities of morphology and distribution of sebaceous glands, but also very commonly with an absence or great reduction in the number of mature hair follicles and, in the presence of ectopic apocrine glands, features not seen in SESD (17). Sebaceous adenoma and sebaceoma are sharply circumscribed multilobular tumors of sebaceous cells usually located in the mid or deep reticular dermis. They lack the plate-like superficial pattern with connections to the epidermis characteristic of SESD, although some authors consider it possible to regard it as a superficial special type of sebaceous adenoma or sebaceoma (1-4, 6-7). Basal cell carcinoma would demonstrate peripheral palisading of basaloid cells, retraction of collagen around tumor nests, numerous mitoses and apoptosis, higher cellularity, and a higher nuclear/cytoplasm ratio $(2-4,6-7,17)$. The sebaceous differentiation is also more limited and less wellorganized in basal cell carcinomas (4). Sebaceous differentiation is usually not seen in seborrheic keratosis, but a few sebocytes scattered throughout the lesion can be seen in rare cases $(2,6,10)$. The tumor of the follicular infundibulum also does not usually have sebaceous differentiation and is attached to the epidermis by thin branching cords of basaloid cells with paler cytoplasm and more prominent peripheral palisading $(1,3,6-7,17)$. As previously stated, a reticulated pattern is occasionally described in SESD, which could be similar to the architecture of the tumor of the follicular infundibulum, and one example of morphological overlap was described as a hybrid of follicular infundibulum tumor and SESD, which is a reminder that a precise classification is not always possible $(8,10-11,16)$.

Initially reported as superficial epithelioma due to its unknown histogenesis and biological behavior, the term has remained unchanged by some authors and has been criticized by others, who have proposed replacing the name with reticulated acanthoma (13). Although we agree that this has the merit of removing the confusing epithelioma term from this benign tumor, we must concur with the opinion of LeBoeuf et al. when they consider the name reticulated acanthoma to be too restrictive (9). It describes a reticular pattern shared by only a few of the reported tumors and which, for example, was not featured in the original lesion described by Rothko et al. or in the case we report. Even more confusing is to only consider as RASD those tumors with a reticular pattern and as SESD those with broader attachment to the epidermis or, as proposed by some authors, to differentiate both entities based on the presence or absence of mature sebaceous ductal structures (8). For the lesion we report, we would rather use the simpler term acanthoma with sebaceous differentiation, without any other sub-classification or division that fails to demonstrate clinical relevance.

In conclusion, SESD is a rare and benign tumor, usually on the face of middle-aged males, with distinctive histological features that allow differential diagnosis. The yet unclear association with Muir-Torre syndrome makes it an important tumor to recognize and to test for microsatellite instability.

\section{References}

1. Rothko K, Farmer ER, Zeligman I. Superficial epithelioma with sebaceous differentiation. Arch Dermatol. 1980;116:329-31.

2. Friedman KJ, Boudreau S, Farmer ER. Superficial epithelioma with sebaceous differentiation. J Cutan Pathol. 1987;14:193-7.

3. Vaughan TK, Sau P. Superficial epithelioma with sebaceous differentiation. J Am Acad Dermatol. 1990;23:760-2.

4. Kato N, Ueno H. Superficial epithelioma with sebaceous differentiation. J Dermatol. 1992;19:190-4.

5. Sánchez Yus E, Requena L, Simón P, Sánchez M. Complex adnexal tumor of the primary epithelial germ with distinct patterns of superficial epithelioma with sebaceous differentiation, immature trichoepithelioma, and apocrine adenocarcinoma. Am J Dermatopathol. 1992;14:245-52.

6. Akasaka T, Imamura Y, Tomichi N, Kon S. A case of superficial epithelioma with sebaceous differentiation. J Dermatol. 1994;21:264-7.

7. Lee MJ, Kim YC, Lew W. A case of superficial epithelioma with sebaceous differentiation. Yonsei Med J. 2003;44:347-50.

8. Fukai K, Sowa J, Ishii M. Reticulated acanthoma with sebaceous differentiation. Am J Dermatopathol. 2006;28:158-61.

9. LeBoeuf NR, Mahalingam M. Acanthomatous superficial sebaceous hamartoma? A study of six cases with clarification of the nomenclature. J Cutan Pathol. 2007;34:865-70.

10. Haake DL, Minni JP, Nowak M, Abenoza P, Nousari CH. Reticulated acanthoma with sebaceous differentiation. Lack of association with Muir-Torre syndrome. Am J Dermatopathol. 2009;31:391-2.

11. Shon W, Wolz MM, Newman CC, Bridges AG. Reticulated acanthoma with sebaceous differentiation: another sebaceous neoplasm associated with Muir-Torre syndrome? Australas J Dermatol. 2014;55:e71-3.

12. Aldrees SS, Zoroquiain P, Logan PT, Qutub MF, Vila N, Bravo-Filho V, et al. Superficial epithelioma with sebaceous differentiation involving the eyelid: a case report. J Med Case Rep. 2014;8:466.

13. Steffen C, Ackerman AB. Neoplasms with sebaceous differentiation. Philadelphia: Lea \& Febiger; 1994.

14. Sáchez Yus E, Requena L, Simón P, del Río E. Sebomatricoma: a unifying term that encompasses all benign neoplasms with sebaceous differentiation. Am J Dermatopathol. 1995;17:213-21.

15. López JC-H, Taldibaeva G, Sperhake K, Park B-H, Stege H, Böer-Auer A. Reticulated acanthoma with apocrine differentiation. J Cutan Pathol. 2011;38:432-4.

16. Mahalingam M, Bhawan J, Finn R, Stefanato CM. Tumor of the follicular infundibulum with sebaceous differentiation. J Cutan Pathol. 2001;28:314-7.

17. Calonje E, McKee PH. McKee's pathology of the skin: with clinical correlations. 4th ed. Edinburgh: Elsevier, Saunders; 2012. 